ISSN 0001-6002/2002/44/2/68-73

A cta M édica Costarricense, $\odot 2002$

Colegio de Médicos y Cirujanos

\title{
Tamizaje de retinopatía diabética en diabéticos tipo 2 en un área de salud del área metropolitana
}

\author{
Adriana Laclé1, Carlos Mora², Manuel García-C³
}

J ustificación: La Diabetes M ellitus tipo 2 es una enfermedad de alta preval encia en el país; alrededor de 130,000 costarricenses la padecen. Sus complicaciones tienen gran impacto en la autonomía de estas personas. U na de sus complicaciones microvasculares es la retinopatía diabética, que si no se diagnostica tempranamente y se le interviene lleva a la pérdida de la visión. Su tratamiento es eficaz, logrando disminuir hasta en dos tercios el riesgo de ceguera, si se siguen dos recomendaciones: hacer un diagnóstico temprano y proporcionando tratamiento con foto-coagulación cuando sea indicado. En Costa Rica no se conoce la prevalencia de esta complicación y tampoco cual es la al ternativa de solución de su atención en nuestro sistema de salud.

Objetivo: Describir cómo se ha enfrentado la búsqueda y atención de la retinopatía diabética en un área de sal ud del Á rea M etropolitana, considerando los diferentes niveles de atención; así como analizar la prevalencia de la retinopatía diabética, después de implementado un tamizaje para su detección.

Método: Se revisaron los expedientes de 572 diabéticos tipo 2 registrados al 31 de diciembre del 2000 en 6 EBAIS del Á rea de Salud 3 de Desamparados, anotándose el resultado del fondo de ojo que no tuviera más de dos años de antigüedad. A los pacientes que carecían de este examen se les contactó y se les realizó el fondo de ojo por personal experimentado.

Resultados: El 93\% de los diabéticos no tenían fondo de ojo en los últimos 2 años independientemente del nivel de atención donde llevara su control. M ás aún, alrededor del $90 \%$ no tenía documentado un fondo de ojo en ningún momento en el expediente. El tamizaje realizado logró aumentar la cobertura de fondo de ojo a un $60 \%$. Se obtuvieron datos de fondo de ojo de 345 pacientes (60.3\%). De éstos, $27.5 \%$ presentaron algún tipo de retinopatía diabética; $16.5 \%$ no proliferativa, $8.7 \%$ no proliferativa severa y un $2.3 \%$ con retinopatía proliferativa - Fueron diagnosticados con el tamizaje 30 pacientes con retinopatía no proliferativa severa que requerían tratamiento con foto-coagulación de inmediato. Tres de esos paciente con edema macular requerían fotocoagulación urgente y se encontró dificultad para referirlos al III nivel de su área de atracción por no haber citas prontas.

Conclusión: El presente estudio en comunidad demostró que no se está detectando la retinopatía diabética por cuanto no se hace fondo de ojo a los pacientes y hay dificultad para su intervención en el III nivel.

El fondo de ojo debe realizarse a todos los diabéticos, no se está realizando y con las condiciones actuales de nuestro Sistema de Salud no se puede pretender que se haga si se delega toda la responsabilidad en atención primaria. Es necesario buscar estrategias para captar el $100 \%$ de la retinopatía diabética y darles tratamiento oportuno. Los expertos sugieren que para lograr una cobertura universal el método de tamizaje debe tener base comunitaria y el punto en donde se realiza debe ser de fácil acceso a la población. Existen varias modalidades pero la estrategia a definir debe hacerse considerando las condiciones locales propias. En Costa Rica es posible lograrlo.

Descriptores: Diabetes mellitus tipo 2, retinopatía diabética, tamizaje, prevalencia

Abreviaturas: Diabetes M ellitus tipo 2 (DM 2), retinopatía diabética (RD), Fondo de ojo (FO), Clínica Dr. Marcial Fallas Díaz(CMF), Hospital Calderón Guardia(HCG) , Hospital Blanco Cervantes (HBC), Hospital San Juan de Dios (HSJD). United Kingdom Prospective Diabetes Study Group (UK PDS)

Instituto de Investigaciones en Salud (INISA -UCR)

M édico Internista, Hospital M éxico

Hospital Calderón Guardia
Recibido: 22 de enero del 2002

Aceptado para publicación: 23 de abril del 2002

Correspondencia: Dra. A driana L aclé, INISA, Universidad de Costa Rica. Fax: 2075130, E-mail: alacle@ cariari.ucr.ac.cr

* Proyecto N o. 742-99-339 Prevalencia, Incidencia e Historia "natural" de la Diabetes tipo 2 en un Á rea de Salud de la M eseta Central. Vicerectoría de Investigación U niversidad de Costa Rica. 
En Costa Rica, la Diabetes mellitus tipo 2 (DM 2) es una patología altamente prevalente (5-6\% mayores de 20 años) y con un alto costo, tanto en la atención médica como en la calidad de vida de los pacientes que la sufren. Se estima que al rededor de 130,000 costarricenses la padecen y solo la mitad lo saben. Es una enfermedad con alta morbimortalidad, debido a sus complicaciones crónicas en cuatro órganos blanco predominantes: corazón, riñón, miembros inferiores y ojos.

La retinopatía diabética (RD) es una de las complicaciones microvasculares de la enfermedad tanto en el tipo 1 como en el tipo 2, que lleva a la pérdida de la visión; es la primera causa de nuevos casos de ceguera en los adultos de 20 a 74 años en países desarrollados ${ }^{1}$.

La prevalencia de la retinopatía diabética está fuertemente relacionada con la duración de la diabetes ${ }^{1,2}$ y de su control metabólico ${ }^{3-6}$. Después de 20 años de evolución de la DM , casi el $100 \%$ de los pacientes tipo 1 y más del $60 \%$ de los pacientes tipo 2 tienen algún grado de retinopatía. En un estudio epidemiológico realizado en Wisconsin ${ }^{2}$ la causa de ceguera en un tercio de los ciegos mayores de 30 años fue secundaria a esta complicación crónica.

En Costa Rica no se tienen datos de prevalencia de la retinopatía diabética; lo poco que se sabe es lo presentado en la Encuesta Nacional de Hogares realizada en ${ }^{1998^{7}}$, en la cual se obtuvo que un $25.5 \%$ de las personas diabéticas mayores de 40 años auto refirieron tener disminución de la vista o ceguera, sin poder precisar si esta limitación física era secundaria a su DM u a otras patologías.

Según la A sociación A mericana de Diabetes y la Academia Americana de Oftalmología si se siguen sus recomendaciones ${ }^{8}$ se puede disminuir en dos tercios el riesgo de ceguera de $28 \%$ a $12 \%$. Estas recomendaciones son dos: realizar a todo diabético tipo 2 en el momento de su diagnóstico un fondo de ojo con dilatación pupilar por un oftalmólogo; luego, anualmente por personal experimentado; y proporcionar tratamiento con fotocoagulación cuando sea necesario.

Es conocido que hasta el $21 \%$ de los DM 2 tienen al gún grado de retinopatía cuando son diagnosticados ${ }^{1,5}$ y que un control metabólico adecuado reduce sustancialmente el riesgo de ceguera, como lo demuestra el estudio del U nited Kingdom Prospective Diabetes Study Group (UK PDS) en la DM 24.5 y el Diabetes Control and Complications Trial (DCCT) en la DM tipo $\mathrm{I}^{3}$. Estos estudios mostraron que un buen control glicémico puede reducir el riesgo de retinopatía proliferativa.

¿Es pertinente realizar el tamizaje para la detección precoz de la DM tipo 2 y de la retinopatía diabética? Cualquier prueba de tamizaje en medicina debe ser realizada cuando los resultados tengan una probabilidad razonable de alterar el tratamiento, cuando el riesgo-beneficio de hacer la prueba es favorable al paciente y cuando el costo-beneficio sea competitivo con otras estrategias de intervención. En este sentido, el tamizaje de la DM 2 y la RD reúne las tres indicaciones anteriores. A demás, no cabe duda que entre más temprano sea realizado el diagnóstico de la DM 2, menos pacientes tendrán RD en el momento de diagnóstico.

Según los datos anteriores, en cualquier país en donde la DM 2 sea prevalente, como es el caso de Costa Rica, es importante implementar un Programa $\mathrm{N}$ acional de Vigilancia E pidemiológica de la Diabetes, iniciándose con su detección precoz, incluyendo como parte de su debido control la detección de la RD; complicación que es capaz de ser sujeta de intervención con muy buenos resultados para los pacientes.

El objetivo del presente trabajo es describir cómo se ha enfrentado la búsqueda y atención de la retinopatía diabética en un área de salud del Á rea M etropolitana, considerando los diferentes niveles de atención; así como analizar la prevalencia de la RD, después de implementado un tamizaje.

\section{Metodología}

Este proyecto fue aprobado por el Consejo Científico del INISA y la Vicerrectoría de Investigación de la Universidad de Costa Rica.

Se hizo la investigación con 572 diabéticos tipo 2, 365 mujeres (63.8\%) y 207 hombres (36.2\%) registrados al 31 de diciembre del 2000, en 6 EBAIS del Área de Salud 3 de Desamparados: Fátima, Patarrá, Río Azul, Linda Vista, Guidos 2 y Guidos 4.

Como norma de atención a los pacientes diabéticos a nivel nacional, está la realización del fondo de ojo anualmente. Es además una variable a evaluar en los compromisos de gestión de las Áreas de Salud. Como apoyo a la atención integral del paciente DM 2 de los EBAIS estudiados y para cumplir con este examen para el año 2001, se revisaron los expedientes en los centros de salud en donde tenían su control para la diabetes o su control oftalmológico, anotándose el resultado del fondo de ojo que no tuviera más de dos años de antigüedad. A los pacientes que carecían de este examen se les contactó. A quellos que aceptaron, se les realizó el fondo de ojo con dilatación de pupila. Éste fue realizado por personal experimentado durante el primer semestre del 2001; en la Consulta Externa del Hospital Calderón Guardia (HCG) por oftalmólogo con el método indirecto y en el Hospital M éxico por un médico internista con el método directo. Cuando existió interrogante sobre la clasificación del grado de retinopatía, se reval oró por medio indirecto en el HCG por el oftalmólogo.

\section{Resultados}

De los 572 diabéticos tipo 2 registrados en los EBAIS por atención primaria, se ubicó en un $94.7 \%$ de ellos el centro en donde llevaban su control (incluye control particular y 
empresa). Veintinueve pacientes (5.1\%) no tenían control alguno.

Solo el $7.3 \%$ tenía un fondo de ojo documentado en el expediente en los últimos dos años o estaban en control oftalmológico por ceguera, glaucoma, retinopatía diabética severa o iridocilitis. El 89\% no tenía registrado examen de fondo de ojo en todo el expediente.

A I final del estudio, se obtuvieron datos de fondo de ojo de 345 pacientes (60.3\%), 220 con el método directo y 125 con el indirecto. Veintiséis pacientes fueron revalorados por el método indirecto para su clasificación final. Noventa y cinco pacientes $(27.5 \%)$ presentaron algún tipo de $R D$; $16.5 \%$ con RD no proliferativa (57 pacientes), $8.7 \%$ con RD no proliferativa severa (30 pacientes) y un $2.3 \%$ con RD proliferativa (8 pacientes). (Cuadro No. 1-3).

El $10.7 \%$ de los pacientes $(n=61)$ tenía algún tipo de control en Hospitales Clase A o Clase B (Hospital Blanco Cervantes). De estos 61 pacientes, sólo el 30\% tenía un fondo de ojo documentado en el expediente en los últimos dos años a diciembre del 2000. Con el estudio, se logró aumentar el porcentaje de pacientes con fondo de ojo a un $51 \%$ (31 pacientes). De éstos, doce presentaron RD (40\%); 4 pacientes con RD proliferativa, de los cuales sólo dos tenían tratamiento de ray os láser. Dos de estos pacientes presentaron ceguera secundaria a la RD.

En el servicio de M edicina Interna de la Clínica Dr. M arcial Fallas $(C M F)$, clínica mayor del área de atracción de los EBA IS en estudio, seguían su control 198 pacientes (34.6\%). De éstos, $94 \%$ no tenían fondo de ojo en los últimos dos años. Por el estudio, se logró realizar el fondo de ojo a 161 pacientes (81.3\%). Se obtuvo un $29.2 \%$ con RD ( $n=47) ; 3$ de ellos tenían retinopatía proliferativa, de los cuales uno no había sido detectado. Del resto, 16 tenían retinopatía no proliferativa severa que requerían rayos láser, solo cuatro estaban en tratamiento oftalmológico.

La gran mayoría del total de pacientes tenía su control en el primer nivel (EBAIS): 253 pacientes (44.2\%). El 95\% de esta población no tenía fondo de ojo registrado en el expediente. A través del estudio, se logró realizar el fondo de ojo a 134 personas $(53 \%$ ) con un porcentaje de RD de $23.1 \%$, de los cuales un paciente tenían RD proliferativa que no había sido detectada, 10 pacientes se les detectó con el tamizaje RD no proliferativa severa que requería tratamiento con láser de inmediato.

Doce de los pacientes $(2.1 \%)$ se controlaban en diferentes clínicas fuera de su área de atracción en atención primaria y trece ( $2.3 \%)$ tenía control de carácter particular o con médico de empresa. Se logró realizar el fondo de ojo a 11 de estos 25 pacientes, encontrándose 2 pacientes con RD no proliferativa y en uno era severa pero ya había recibido tratamiento con láser.

Veintinueve pacientes $(5.1 \%)$ no tenían control alguno y se logró realizar fondo de ojo a 8 de ellos, encontrándosele RD no proliferativa severa a dos, los cuales necesitaban tratamiento de láser inmediato.

De los 95 pacientes con RD, 8 pacientes (8.4\%) presentaron retinopatía proliferativa, $30 \mathrm{RD}$ no proliferativa severa que requerían tratamiento con láser (31.6\%) y el resto (60\%) RD Base. Dos pacientes con RD proliferativa no habían recibido tratamiento, cuatro tenían ceguera secundaria a desprendimiento de retina y solo dos se habían beneficiado de el láser terapia. De los 30 con RD severa solo cuatro habían recibido tratamiento con láser, el resto fue diagnosticado por el tamizaje.

\begin{tabular}{|c|c|c|c|c|c|c|}
\hline \multicolumn{7}{|c|}{$\begin{array}{l}\text { Cuadro No. } 1 \\
\text { Distribución porcentual de los pacientes estudiados según centro de salud de control y } \\
\text { resultados de fondo de ojo }\end{array}$} \\
\hline $\begin{array}{l}\text { Centro de } \\
\text { salud en que } \\
\text { sigue control }\end{array}$ & $\begin{array}{l}\text { Pacientes } \\
\text { Registrados } \\
\text { a Dic } 2000\end{array}$ & $\begin{array}{l}\text { \% según } \\
\text { centro de } \\
\text { salud }\end{array}$ & $\begin{array}{l}\text { No. Pacientes } \\
\text { con fondo } \\
\text { de ojo }\end{array}$ & $\begin{array}{l}\% \text { Con fondo } \\
\text { de ojo }\end{array}$ & $\begin{array}{l}\% \text { Sin } \\
\text { retinopatía }\end{array}$ & $\begin{array}{l}\% \text { Con } \\
\text { retinopatía }\end{array}$ \\
\hline EBAIS & 253 & 44.2 & 134 & 53.0 & 76.9 & 23.1 \\
\hline CMF & 198 & 34.6 & 161 & 81.3 & 70.8 & 29.2 \\
\hline HSJD & 36 & 6.3 & 17 & 47.2 & 58.8 & 41.2 \\
\hline $\mathrm{HBC}$ & 13 & 2.3 & 9 & 69.2 & 66.7 & 33.3 \\
\hline HCG & 9 & 1.6 & 3 & 33.3 & 66.7 & 33.3 \\
\hline H. MEXICO & 3 & 33.3 & 2 & 66.7 & 50.0 & 50.0 \\
\hline Otras clínicas mayores & 12 & 2.1 & 5 & 41.0 & 80.0 & 20.0 \\
\hline otros & 13 & 2.3 & 6 & 46.2 & 83.3 & 16.7 \\
\hline Sin control & 29 & 5.1 & 8 & 27.6 & 62.5 & 37.5 \\
\hline Sin ubicar & 6 & 1.0 & 0 & 0.0 & 0.0 & 0.0 \\
\hline TOTAL & 572 & 100 & 345 & 60.3 & $72.5(250)$ & $27.5(95)$ \\
\hline
\end{tabular}

70 AMC, abril-junio 2002, vol 44 (2) 


\begin{tabular}{|c|c|c|c|c|c|c|c|}
\hline \multicolumn{8}{|c|}{$\begin{array}{c}\text { Cuadro No. } 2 \\
\text { Tipo de Retinopatía Diabética según centro de control }\end{array}$} \\
\hline $\begin{array}{l}\text { Centro de } \\
\text { salud } \\
\text { de control }\end{array}$ & No. Pacientes & $\begin{array}{l}\text { Pacientes } \\
\text { con fondo } \\
\text { de ojo }\end{array}$ & $\begin{array}{c}\text { Pacientes } \\
\text { con } \\
\text { Retinopatía }\end{array}$ & $\begin{array}{c}\text { RD NO } \\
\text { proliferativa } \\
\text { severa* }^{*}\end{array}$ & $\begin{array}{l}\text { Pacientes } \\
\text { con RD } \\
\text { proliferativa }\end{array}$ & $\begin{array}{c}\% \text { con RD } \\
\text { que ameritaba } \\
\text { láser terapia }\end{array}$ & $\begin{array}{l}\text { Retinopatía } \\
\text { proliferativa } \\
\text { con ceguera }\end{array}$ \\
\hline I Nivel & 265 & 139 & 32 & 10 & 1 & 34.4 & 1 \\
\hline II Nivel & 198 & 161 & 47 & 16 & 3 & 40.4 & 1 \\
\hline III Nivel & 61 & 31 & 12 & 1 & 4 & 41.7 & 2 \\
\hline Otros & 13 & 6 & 1 & 0 & 0 & 0.0 & 0 \\
\hline Sin control & 29 & 8 & 3 & 2 & 0 & 66.7 & 0 \\
\hline Sin ubicar & 6 & 0 & 0 & 0 & 0 & 0.0 & 0 \\
\hline TOTAL & 572 & 345 & 95 & 30 & 8 & 40.0 & 4 \\
\hline
\end{tabular}

\begin{tabular}{|c|c|c|c|c|c|c|c|c|c|c|}
\hline \multirow{3}{*}{$\begin{array}{l}\text { Años de } \\
\text { evolución }\end{array}$} & \multicolumn{8}{|c|}{$\begin{array}{c}\text { Cuadro No. } 3 \\
\text { Tipo de Retinopatía Diabética según años de evolución }\end{array}$} & & \\
\hline & \multicolumn{2}{|c|}{$\begin{array}{c}\text { No. de } \\
\text { pacientes con } \\
\text { fondo de ojo }\end{array}$} & \multicolumn{2}{|c|}{$\begin{array}{c}\text { Con } \\
\text { Retinopatía } \\
\text { Diabética }\end{array}$} & \multicolumn{2}{|c|}{$\begin{array}{l}\text { Con RD no } \\
\text { proliferativa }\end{array}$} & \multicolumn{2}{|c|}{$\begin{array}{c}\text { Con RD no } \\
\text { proliferativa } \\
\text { SEVERA }\end{array}$} & \multicolumn{2}{|c|}{$\begin{array}{l}\text { CON RD } \\
\text { proliferativa }\end{array}$} \\
\hline & $\mathrm{n}$ & $\%$ & $\mathrm{n}$ & $\%$ & $\mathrm{n}$ & $\%$ & $n$ & $\%$ & $\mathrm{n}$ & $\%$ \\
\hline $1-4$ & 128 & 37.1 & 11 & 8.6 & 9 & 7.0 & 2 & 1.6 & 0 & 0.0 \\
\hline $5-9$ & 88 & 25.5 & 16 & 18.2 & 13 & 14.8 & 3 & 3.4 & 0 & 0.0 \\
\hline $10-19$ & 98 & 28.4 & 49 & 50.0 & 25 & 25.5 & 20 & 20.4 & 4 & 4.1 \\
\hline 20 y más & 31 & 9.0 & 19 & 61.3 & 10 & 32.3 & 5 & 16.1 & 4 & 12.9 \\
\hline TOTAL & 345 & 100 & 95 & 27.5 & 57 & 16.5 & 30 & 8.7 & 8 & 2.3 \\
\hline
\end{tabular}

Catorce pacientes reportaron ceguera, uno de ellos en el ojo izquierdo secundario a un accidente, otro por estrabismo congénito, seis por glaucoma y dos en que no se documentó la causa, aunque uno era portador de un mal perforante plantar; cuatro de ellos por causa de la retinopatía diabética en su mayoría por desprendimiento de retina que corresponde a menos del $1 \%$ del total de pacientes del estudio.

M ás de la mitad de los pacientes tenían menos de 10 años de evolución (62.6\%); entre 10 y 19 años el $28.4 \%$, y más de 20 años sólo 31 pacientes $(9.0 \%)$. A mayor años de evolución, mayor prevalencia de RD, mostrando una relación altamente significativa, con un chi-cuadrado de tendencias en 81.8 con una $p<0.00000$.

\section{Discusión}

La Diabetes Mellitus tipo 2 es una enfermedad crónica prototipo con una alta prevalencia en el país. Sus complicaciones tienen gran impacto en la autonomía de las personas que la padecen. El diagnóstico temprano de la enfermedad, los años de evolución y su control metabólico determinan la severidad y cuan temprano se inician las complicaciones.
La retinopatía diabética es una complicación que si no se trata oportunamente lleva a ceguera. Varios estudios incluyendo el UKPDS documentan que está presente al momento del diagnóstico en el $21 \%$ de los individuos con DM $2^{4}$.

La detección temprana de la RD permitiría medidas terapéuticas como la foto coagulación con láser que podría disminuir las complicaciones que llevan a la ceguera como el desprendimiento de retina.

El presente estudio en comunidad demostró que no se está detectando la RD por cuanto no se hace fondo de ojo (FO) a los pacientes. El 93\% de los diabéticos no tenían examen de FO en los últimos 2 años independientemente del centro donde llevara su control. Más aún, alrededor del $90 \%$ no tenía documentado un $\mathrm{FO}$ en ningún momento en el expediente.

El tamizaje realizado logró aumentar la cobertura de $\mathrm{FO}$ a un $60 \%$. A unque ubicados la mayoría de los pacientes, no fue posible realizarles $\mathrm{FO}$ a un $40 \%$ por imposibilidad física a movilizarse, por su horario laboral y por la falta de recurso económico para transportarse al nivel III ( hospitalario).

La prevalencia de RD fue de $27.2 \%$ en forma general para esta comunidad. En la literatura universal la prevalencia 
varía desde una mínima como en el sur de India con un $1.78 \%{ }^{10}$, como en Mauritania con una variabilidad importante entre etnias y un promedio de $14.8 \%^{11}$ hasta un $33 \% \%$ en DM 2 en un estudio en Alberta, Canadá ${ }^{22}$. El promedio ronda en un $25 \%$ en general, y aumenta consi derablemente según los años de evolución hasta un $60 \%$ en personas con mas de 20 años de evolución ${ }^{1,13}$. Esto último correlaciona bien con los datos obtenidos en este estudio, en que a más años de evolución mayor porcentaje de RD y mayor la severidad, obteniéndose un $61 \%$ con RD en las personas con más de 20 años de evolución. (Cuadro No. 4). La prevalencia de RD proliferativa $(2.3 \%)$ fue menor que lo reportado mundialmente, que usualmente es del orden del $5 \%{ }^{1,11,12}$. De estos pacientes, solo la mitad habían recibido láser terapia, aunque todos ya ciegos, reflejando ello que muy probablemente fueron captados tardíamente. Otros dos pacientes eran conocidos pero no recibían tratamiento oftalmológico alguno. El tamizaje detectó 2 casos más de RD proliferativa para un total de 8 pacientes.

Fueron diagnosticados con el tamizaje 30 pacientes con RD no proliferativa severa que requerían tratamiento con foto coagulación de inmediato. Tres de esos paciente con edema macular requerían igualmente foto coagulación urgente. Estos pacientes que requerían tratamiento oftalmológico pronto, han encontrado citas a largo plazo, con el agravante que el Hospital tipo A de su área de atracción actualmente carece de láser terapia y deben referirlos a otro Hospital tipo A, reflejando con ello la poca capacidad resolutiva del III nivel con esta patología que crece día a día.

Al analizar el porcentaje de RD por nivel de atención se encuentra que en el III nivel hay mayor prevalencia (38.7\%) y menor en el nivel I (23\%), con un porcentaje intermedio $(29.2 \%)$ en el nivel II; lo cual es esperable por la referencia de pacientes complicados a niveles superiores de atención.

En el año 1994, la C.C.S.S. editó y distribuyó en todo el país el $M$ anual para el tratamiento de la D.M. no insulino dependiente, en donde se incluían las normas que regirían la atención de estos pacientes. Se estipulaba en el protocolo de control clínico la realización de FO en la primera visita y anualmente. Esta investigación documentó que estos lineamientos en esta área de salud y también en sus centros de referencia de nivel II y III distan mucho de la normativa. A nivel primario varias causas podrían ser mencionadas como razones para no practicar el FO: 1. No equipamiento de los centros, 2. Condiciones físicas no adecuadas de los establecimientos. 3. Poca disponibilidad de tiempo en la consulta médica 4. Imposibilidad de dilatar pupila. 5. Impericia. Con relación a los equipos diagnósticos, en los 2 últimos años, la mayoría de los centros tienen equipo adecuado. En cuanto a tiempo en la consulta general normal que en promedio es de 10 minutos ( 5 pacientes por hora) es difícil realizarlo. La dilatación conlleva peligros inminentes que la mayoría de los centros no están en capacidad de abordar. Un fondo de ojo sin dilatación, sin oscuridad y con falta de pericia tiene poca utilidad. ${ }^{14,15}$ Se sabe que en una consulta general, el médico tiene poca posibilidad de mantenerse entrenado en el diagnóstico del FO. La A sociación A mericana de Oftal mología sugiere que el primer FO al momento del diagnóstico se realice por un oftalmólogo y luego anualmente por personal experimentado.

A I momento actual, aunque el $\mathrm{FO}$ debe realizarse a todos los diabéticos, no se está realizando y con las condiciones actuales de nuestro Sistema de Salud no se puede pretender que se haga si se delega toda la responsabilidad en la atención primaria. Es necesario buscar estrategias para captar el $100 \%$ de la RD y darles tratamiento oportuno. Los expertos sugieren que para lograr una cobertura universal el método de tamizaje debe tener base COMUNITARIA y el punto en donde se realiza debe ser de fácil acceso a la población. Hay muchas modalidades para enfrentar este reto, como podría ser un Programa Nacional de Tamizaje de RD como existen en otros países como en el Reino Unido. Otra de tantas modalidades es la fotografía de retina móvilit en el cual se utiliza un técnico y es a nivel comunitario y también está la angiografía que es más sensible aunque más costosa. Existen varias modalidades pero la estrategia a definir debe hacerse considerando las condiciones locales propias. En Costa Rica es posible lograrlo.

\section{Abstract}

Costa Rica has a population of 4 million and an estimated prevalence of Diabetes mellitus type 2 of $6 \%$. This represents around 130,000 diabetic patients in the whole country. It is known that the chronic complications of diabetes have a great impact in the quality of life of these persons. Diabetic rethinopathy is one of the most important; if it is not diagnosed early and treatment, it can lead to loss of vision. The prevalence of this pathology and how well it is approached by the Costa Rican Health System is unknown. The objective of this study is to find out how the Health A rea of Desamparados 3, an urban-marginal comunity of the metropolitan area, has coped with this complication up to now, and to determine with a retinopathy screening intervention an estimate of its prevalence.

The medical records of 572 diabetics where checked, searching for the results of eye fundoscopic examination in the last two years. Those patients that had no previous eye examination were dilated and examined by experience clinicians. Ninety three percent of the patients had had no eye test in the last two years, even worse, $89 \%$ had no eye examination in their medical records at all. After the screening intervention, a funduscopic exam was obtained in 345 patients $(60.3 \%)$. Of these, $27 \%$ had diabetic retinopathy; $16.5 \%$ had basic non proliferative, $8.7 \%$ had severe non proliferative and $2.3 \%$ had with proliferative retinopathy. Thirty patients had severe non proliferative retinopathy that needed fotocoagulation intervention on urgent basis. Three had macular oedema that needed urgent treatment. A ccess to hospitals oftalmologic facilities for the studied area was limited, having waiting lists up to three months. 
This study showed that diabetic retinopathy is not being diagnosed because ophtalmics funduscopic examination is not being done, not only in primary care but also in patients that are controled at hospital level. The screening intervention was well accepted by the patients, but the referral of these patients to the hospitals is not well coordinated.

To achieve near universal coverage for patiets withs this complication, the screening method has to be community based and the point of delivery within easy reach of the population. M any different modalities of screening are in use, depending on local availability of facilities. Costa Rica has to seek the best way to achieve total coverage, and we believe its has the capacity of doing so.

\section{Agradecimientos}

A todos los pacientes diabéticos del Area de Salud Desamparados 3, que con mucho interés por su salud, hicieron el gran esfuerzo económico y de tiempo para trasladarse hasta los Hospitales Clase A para realizarse el Fondo de Ojo.

\section{Referencias}

1. A iello LP, Gardner TW, King GL, Blankenship G, Cavallerano JD, Ferris FL 3rd, et al, Diabetic Retinopathy ( Technical Review). Diabetes Care 1998, 21:143-156.

2. Klein R., Klein B.E., M oss, S.E., Davis, M.D., and DeM ets, D.L. The Wisconsin Epidemiologic Study of Diabetic Retinopathy. III. Prevalence and risk of diabetic retinopathy when age at diagnosis is 30 years or older. A rch. O phthalmol, 1984,102: 527-532.

3. Diabetes Control and Complications Trial Research Group: Progression of retinopathy with intensive versus conventional treatmente in the DCCT. O phthalmology 1995, 102: 647-661.

4. UK Prospective diabetes Study Group: Intensive blood-glucose control with sulphonylureas or insulin compared with conventional treament and risk of complications in patients with type 2 diabetes (UK PDS 33 ) Lancet 1998, 352:837-853

5. A merican Diabetes A sociation Working group. Implications of the United Kingdom Prospective Diabetes Study. Diabetes Care January 2000 Suplement I :527-531.

6. Ohkubo $\mathrm{Y}, \mathrm{K}$ ishikawa $\mathrm{H}$, A raki E, M iyata $\mathrm{T}$, Isami $\mathrm{S}$, M otoyoshi $\mathrm{S}$. et al, Intensive insulin therapy prevents the progression of diabetic microvascular complications in japanese patients with non-insulindependent diabetes: a randomized prospective 6 -years study. Diabetes Res Clin Pract 1995, 28:103-117.

7. Morice A, Rosello M, A rauz AG, Sánchez G, Padilla G. Diabetes mellitus en Costa Rica: un análisis interdisciplinario. Serie de documentos técnicos, IN CIENSA, Tres Rios, Costa Rica,1999.

8. American College of Physicians, American Academy of Ophtalmology: Screening guidelines for diabetic retinopathy . Clinical guideline, Ophthalmolgy 1992, 99:1626-1628.

9. Diabetic Retinopathy Study Research Group: Indications for photocoagulation treatment of diabetic retinopathy ( DRS Report No 14 ) Int Ophthalmol Clin 1987 27: :239-253.
10. Dandona L., Dandona R., Naduvilath TJ., M CCarty CA. Rao GN. Population based assessment of diabetic retinopathy in an urban population in southern India B ritish J ournal of Ophthalmology. 1999, 83(8):937-940.

11. Dowse GK, Humphrey AR, Collins VR, Plehwe W, Gareeboo H, Fareed $D$ et al, Prevalence and Risk Factors for Diabetic Retinopathy in the Multiethnic Population of Mauritius., Am J Epidemiol 1998;147:448-57.

12. Meng-Hee Tan, MacLean, David $\mathrm{R}$ The prevalence of diabetic retinopathy in southern A Iberta, Canada Clin Invest M ed 1995; 18 (4): 240-246.

13. Stefaniotou M, Kitsos G, Paschides C, Kalogeropoulos Chr., A spiotis $M$, Gorgolis S, et al, The prevalence of Diabetic Retinopathy in type II Diabetes Mellitus in Northwest Greece (Epirus) Greek Annals of Ophthalmology 1997, 1: 65-70.

14. Reenders $\mathrm{K}$, de N obel $\mathrm{E}$, van den $\mathrm{H}$ oogen $\mathrm{H}$, van Weel $\mathrm{C}$. Screening for diabetic retinopathy by general practitioners. Scand J Prim Health Care 1992 Dec;10(4):306-9.

15. Sullivan F.M. Stearn R and MacCuish A.C. The role of general practitioners in diabetic eye care in Lankarshire Diabetic Medicine 1994,13(5) :583-585..

16. Taylor R. Practical community screening for diabetic retinopathy using th mobile retinal camera : report of a 12 centre study. B ritish Diabetic A ssociation M obile Retinal Screening Group. Diabetic M edicine 1996, 13 (11) : 945-52. 